\title{
Efectividad Clínica y Radiográfica de la Pasta Antibiótica CTZ en Pulpotomías de Molares Primarios. Ensayo Clínico Aleatorio Controlado
}

\author{
Clinical and Radiographic Effectiveness of Antibiotic Paste CTZ in \\ Primary Molars Pulpotomy. Ramdomized Controlled Clinical Trial
}

\begin{abstract}
Jesús Luengo Fereira*; Adrián Ramos Medina*; Martha Elena Hernández Montoya*; Cristal Yurixie Díaz Rosas*; Luz Elena Carlos Medrano* \& Iovanna Toscano García*
\end{abstract}

LUENGO, F. J.; RAMOS, M. A.; HERNÁNDEZ, M. M. E.; DÍAZ, R. C. Y.; MEDRANO, L. E. C. \& TOSCANO, G. I. Efectividad clínica y radiográfica de la pasta antibiótica CTZ en pulpotomías de molares primarios. Ensayo clinico aleatorio controlado. Int. J. Odontostomat., 10(3):425-431, 2016.

RESUMEN: El objetivo de este trabajo fue evaluar clínica y radiográficamente la efectividad de la pasta antibiótica CTZ en pulpotomías de molares primarios. Se realizó un ensayo clínico aleatorio controlado, en 40 molares primarios de 40 preescolares con edades entre 3 y 6 años. Los pacientes fueron seleccionados y asignados aleatoriamente a dos grupos: Formocresol $(n=20)$, CTZ (Cloranfenicol-Tetraciclina-Oxido de Zinc Eugenol, $n=20)$. Los dientes fueron restaurados con ionómero de vidrio y coronas metálicas de acero preformadas. La evaluación clínica y radiográfica fue realizada a los 3 y 6 meses. Se utilizó el programa SPSS v.17 para el análisis de los datos, y la aplicación del Test Exacto de Fisher al $5 \%$. El comportamiento clínico a los 3 meses mostró $75 \%$ de éxito para los molares tratados con formocresol y $70 \%$ para el CTZ. Radiográficamente se obtuvo $90 \%$ de éxito para el grupo con formocresol y $100 \%$ para la pasta CTZ. A los 6 meses el éxito clínico del formocresol fue de $85 \%$ y del CTZ $80 \%$. El éxito radiográfico mostrado fue $65 \%$ para los atendidos con formocresol y $80 \%$ para el CTZ ( $p>0,05)$. No se observaron diferencias significativas entre los grupos de tratamiento. La pasta CTZ es una alternativa en el tratamiento de pulpotomías de molares temporales. Ofrece un efecto antimicrobiano, estabilización del proceso de reabsorción radicular, sin ocasionar daños a la formación del diente permanente.

PALABRAS CLAVE: molares primarios, pulpotomía, formocresol, CTZ.

\section{INTRODUCCIÓN}

La dentición decidua es de fundamental importancia para la fonación, masticación, estética y bienestar psicosocial de los infantes, además ella mantiene el espacio para la erupción favorable de los dientes permanentes. Por lo que, se deben emplear todos los recursos disponibles para evitar su pérdida prematura (Sousa et al., 2014).

No obstante, la prevalencia de caries dental en la dentición primaria sigue manteniendo elevados niveles en la población infantil en México, a pesar de los esfuerzos y avances que se han realizado en materia de promoción de la salud bucal (Molina-Frechero et al., 2015). Está situación incrementa las posibilidades de afectación pulpar por la presencia de caries pro- fundas, conllevando a la pulpa a un estado irreversible, implicando la necesidad de realizar un tratamiento pulpar (Passos et al., 2008) para mantener su integridad y la posibilidad de preservarlos hasta su exfoliación fisiológica (Sousa et al.).

A pesar de lo anterior, la compleja anatomía de los dientes primarios (Subramaniam et al., 2011), aunado a la gama de materiales y fármacos que existen en la actualidad para el tratamiento pulpar y a la dificultad de los clínicos para realizar un diagnostico certero, ha favorecido el uso de pastas antibióticas como una alternativa dentro de la terapia endodóntica, principalmente por su capacidad antimicrobiana, disminución en los costos, rápida aplicación ameritando poco 
LUENGO, F. J.; RAMOS, M. A.; HERNÁNDEZ, M. M. E.; DÍAZ, R. C. Y.; MEDRANO, L. E. C. \& TOSCANO, G. I. Efectividad clínica y radiográfica de la pasta antibiótica CTZ en pulpotomías de molares primarios. Ensayo clinico aleatorio controlado. Int. J. Odontostomat., 10(3):425-431, 2016.

tiempo operatorio, además, puede indicarse en pacientes poco colaboradores (Pinky et al., 2011) e independientemente del diagnóstico pulpar (de Oliveira et al., 2006).

Esta evidencia, ha permitido que desde varios años en la Clínica Integral de la Especialidad en Odontopediatría de la Universidad Autónoma de Zacatecas, se utilice la técnica de pulpotomía a través del uso de la pasta antibiótica CTZ en órganos dentales primarios con afectación pulpar y/o pronóstico dudoso. La pasta CTZ fue descrita en 1959 por Sollier y Cappiello, para el tratamiento de molares temporales con comprometimiento pulpar (Sousa et al.; Passos et al.; Perona et al., 2014). En su composición se encuentra el Cloranfenicol $500 \mathrm{mg}$, Tetraciclina $500 \mathrm{mg}$, Óxido de Zinc 1000 mg. y Eugenol (1 gota) (Perona et al.); siendo los dos últimos adicionados durante el acto operatorio (Sousa et al.).

A pesar del éxito clínico conocido de la técnica de endodoncia convencional con formocresol y a través del uso de otros materiales, la evidencia sobre el uso de las pastas antibióticas en el tratamiento de pulpotomías de molares primarios con vitalidad pulpar, es limitada hoy en día; existiendo la necesidad de desarrollar estudios clínicos con el fin de verificar los resultados clínicos y radiográficos de los dientes primarios tratados bajo este enfoque. El propósito del presente estudio fue evaluar clínica y radiográficamente la efectividad del uso de la pasta antibiótica CTZ en el tratamiento de pulpotomías vitales de molares primarios.

\section{MATERIAL Y MÉTODO}

Población de estudio: El estudio contó con la aprobación del Comité de Bioética del Área de Ciencias de la Salud de la Universidad Autónoma de Zacatecas. Se seleccionaron 40 niños, de ambos sexos, entre 3 y 6 años de edad, cursantes de preescolar del municipio de Calera de Víctor Rosales, Zacatecas, México.

Para la inclusión se consideraron molares con signos clínicos de caries profunda, con vitalidad pulpar, sin tratamiento previo, con evidencia radiográfica de longitud radicular mayor a la mitad (60\%) y sin imágenes de signos patológicos, exposición y sangrado de la cámara pulpar de aspecto normal, con una coloración rojo intenso, con un tiempo de control de la hemorragia no mayor a 5 minutos (con el uso de suero fisiológico). Solo entonces el tejido pulpar en los conductos radiculares fue asumido como normal.

Se excluyeron pacientes con signos y síntomas clínicos y/o radiográficos de degeneración pulpo-radicular; historia de dolor espontáneo, a la palpación, a los cambios térmicos, a la masticación; con algún signo de inflamación, edema, enrojecimiento, calor, rubor, absceso localizado, presencia de botón fistuloso, cámara pulpar con olor fétido y/o exudado purulento, movilidad patológica, evidencia de celulitis, sangrado oscuro-espeso. Radiográficamente: imagen radiolúcida en la región periapical y/o interradicular, signos de reabsorción dentinaria interna o externa, radiolucencia de la furca. Pacientes con enfermedades sistémicas y no colaboradores.

El representante legal recibió un informe detallado y firmó una hoja de consentimiento para autorizar su participación en el estudio, de acuerdo con los principios de la declaración de Helsinki (World Medical Association, 2013).

Diseño experimental: Se realizó un ensayo clínico aleatorio controlado y grupos paralelos. Se realizó un muestreo no probabilístico por conveniencia. Los pacientes seleccionados fueron asignados aleatoriamente a dos grupos de tratamiento; Grupo experimental: pasta antibiótica CTZ (Cloranfenicol, Tetraciclina, Oxido de Zinc Eugenol, n=20); Grupo control: formocresol (Solución de Buckley al $20 \%$, $n=20)$.

Procedimientos Clínicos: Un odontólogo entrenado, realizó todas las pulpotomías en una sola sesión, empleando formocresol y CTZ (fórmula en polvo con $500 \mathrm{mg}$ deTetraciclina, $500 \mathrm{mg}$ de Cloranfenicol y ZOE, mezclados con eugenol en una proporción 1:1:2), según la asignación aleatoria del material, bajo aislamiento absoluto, colocación de anestesia infiltrativa o troncomandibular según el caso (scandonest al $2 \%$ ). La manipulación de los materiales fue realizada por otro odontólogo entrenado.

Para ambos grupos, el acceso a la cámara pulpar y el tejido cariado fue removido con cucharillas de dentina esterilizadas, \#17 y \#18 Hu-Frieddy ${ }^{\circledR}$. Seguidamente, se removió el techo de la cámara pulpar con fresas de carburo redondas \#5, para alta velocidad y esterilizadas. Posteriormente, se amputó la pulpa coronaria con cucharillas de dentina esteri- 
lizadas y se realizó un desgaste compensatorio con fresa Endo-Z ${ }^{\circledR}$ Dentsply USA. El sangrado ocasionado durante el acto operatorio, se controló por medio de abundante irrigación con suero fisiológico, empleando jeringas desechables de $10 \mathrm{~mL}$ y luego se secó con torundas de algodón estériles.

Técnica con pasta antibiótica CTZ: La manipulación de la pasta antibiótica CTZ fue realizada siguiendo las instrucciones de la bibliografía (GonzálezNúñez et al., 2010). Una vez eliminada la totalidad de la pulpa cameral y controlado el sangrado, la pasta antibiótica CTZ fue empacada en el fondo de la cámara pulpar (grosor aproximado $2 \mathrm{~mm}$ ), posteriormente se colocó cemento de IRM ${ }^{\circledR}$ (Dentsply-USA) y se obturó con ionómero de vidrio (Ketac Molar ${ }^{\circledR} 3 \mathrm{M}$ ESPE).

Técnica con formocresol: Una vez controlado el sangrado, se colocó una torunda de algodón estéril impregnada con formocresol Viarden ${ }^{\circledR}$, durante 3 minutos. Fijado el tejido pulpar, se colocó IRM ${ }^{\circledR}$ (Dentsply-USA) y se empacó en el fondo de la cámara pulpar, luego se aplicó ionómero de vidrio (Ketac Molar ${ }^{\circledR}$ 3M ESPE).

Quince días posteriores al tratamiento, los pacientes fueron observados para corroborar la ausencia de signos clínicos patológicos. Seguidamente se les colocó en los molares atendidos coronas metálicas de acero (3M Dental Products ${ }^{\circledR}$, USA), cementadas con ionómero de vidrio $\left(\mathrm{Ketac}^{\circledR}, 3 \mathrm{M}\right.$ ESPE AG, Germany). La oclusión fue revisada y ajustada.

Evaluación: Las evaluaciones clínicas y radiográficas fueron realizadas a los 3 y 6 meses, por una Odontopediatra que no intervino en la ejecución de los procedimientos y sin conocer el tipo de tratamiento realizado en cada uno de los molares.

- Éxito clínico, ausencia de cualquier condición fuera de lo normal. Fracaso se consideró, la presencia de dolor, signos de inflamación, presencia o ausencia de fistula, cicatrización de fistula, absceso, exudado purulento, olor fétido, celulitis, movilidad dental patológica, el estado de los tejidos periodontales y otro (otra condición no descrita anteriormente).

- Éxito radiográfico, ausencia de imágenes que indicaran la presencia de cualquier condición fuera de lo normal. Fracaso, presencia de absceso periodontal y/o periapical, lesión en furca, pérdida de trabécula ósea, reabsorción radicular externa o interna, engrosamiento irregular del ligamento periodontal, otra (otra condición, no incluida dentro de las anteriores, observada por el evaluador).

Análisis estadístico: El procesamiento estadístico se realizó con la aplicación SPSS-Windows V17.0 (SPSS, Inc, Chicago IL). Para el análisis comparativo se utilizó el Test Exacto de Fisher, a un nivel de significancia del $5 \%$.

\section{RESULTADOS}

Luego de 6 meses de evaluación, la totalidad de los pacientes culminaron el estudio, distribuyéndose en $19(48,5 \%)$ del sexo femenino y $21(52,5 \%)$ del sexo masculino.

La evaluación de los 3 meses refleja el comportamiento clínico de los grupos de tratamiento, observándose que los molares tratados dentro del grupo control mostraron un mayor porcentaje de éxito ( $75 \%$ ), frente a los molares del grupo experimental (70\%), sin embargo estas diferencias no fueron estadísticamente significativas $(p>0,05)$. En cuanto a los fracasos clínicos seis correspondieron al grupo experimental, teniendo un molar con movilidad, uno con cicatrización de fistula y cuatro con enrojecimiento e inflamación de la encía. En el grupo control, se presentaron 5 casos, dos molares con movilidad dental y tres con enrojecimiento e inflamación de la encía (Tabla I).

Tabla I. Comportamiento clínico a los 3 meses de evaluación según tipo de tratamiento.

\begin{tabular}{llcccccc}
\hline & & \multicolumn{2}{c}{ Éxito } & \multicolumn{2}{c}{ Fracaso } & \multicolumn{2}{c}{ Total } \\
\cline { 3 - 7 } & & $\mathbf{n}$ & $\%$ & $\mathbf{n}$ & $\%$ & $\mathbf{n}$ & $\%$ \\
Tipo de tratamiento & $\mathbf{C T Z}$ & 14 & 70 & 6 & 30 & 20 & 50 \\
& Formocresol & 15 & 75 & 5 & 25 & 20 & 50 \\
Total & & 29 & 72.5 & 11 & 27,5 & 40 & 100 \\
\hline
\end{tabular}

Test de Fisher: 0,499 $(p<0,05)$. 
LUENGO, F. J.; RAMOS, M. A.; HERNÁNDEZ, M. M. E.; DÍAZ, R. C. Y.; MEDRANO, L. E. C. \& TOSCANO, G. I. Efectividad clínica y radiográfica de la pasta antibiótica CTZ en pulpotomías de molares primarios. Ensayo clinico aleatorio controlado. Int. J. Odontostomat., 10(3):425-431, 2016.

En relación a la evaluación radiográfica (Tabla II), todos los molares tratados con la pasta antibiótica CTZ no mostraron signos de lesión, mientras que los atendidos con formocresol obtuvieron el $90 \%$ sin lesión aparente. Solo dos molares primarios del grupo control, presentaron una evidente afectación, con absceso periodontal $(p<0,05)$.

En la Tabla III, se presenta una relación de los hallazgos clínicos y radiográficos para ambos grupos, siendo que para el grupo experimental el éxito clínico fue del $70 \%$ y el fracaso clínico del $30 \%$, sin evidencia de fracaso radiográfico ni fracaso clínico radiográfico. Por otro lado, en el grupo control el $75 \%$ corresponde al éxito clínico del tratamiento y $25 \%$ al de fracasos clínicos (10\% radiográficos y $10 \%$ clíni$\cos$ - radiográficos $),(p<0,05)$.

Para la evaluación de los 6 meses (Tabla IV), puede observándose que los molares tratados con formocresol mantuvieron un $85 \%$ de éxito clínico, mayor al $80 \%$ de los molares atendidos con la pasta antibiótica CTZ $(p ? 0,05)$. Entre los fracasos clínicos se encontraron en el grupo experimental: presencia de fistula, dos cicatrizaciones de fistula y un enrojecimiento de la encía. En el grupo control, se presentaron dos presencias de fistulas y una movilidad dental.

Conforme a la evaluación radiográfica (Tabla V), los molares tratados con la pasta antibiótica CTZ mostraron $80 \%$ de éxito, en comparación a los molares tratados con formocresol $65 \%(p<0,05)$. De igual manera los fracasos radiográficos observados en el grupo experimental ( $20 \%$ ) fueron menores que en el control (35\%), correspondiendo en todos los casos a la presencia de abscesos periodontales.

En la Tabla VI, se relacionan los hallazgos clínicos y radiográficos para ambos grupos. Donde el éxito clínico para el grupo experimental fue del $80 \%$, el fracaso clínico y el radiográfico del $20 \%$, el clínico radiográfico del $5 \%$. Por otro lado, en el grupo control el $85 \%$ corresponde al éxito clínico del tratamiento, $15 \%$ al de fracasos clínicos, $35 \%$ al fracaso radiográfico y $5 \%$ clínicos - radiográficos $(p<0,05)$.

Tabla II. Comportamiento radiográfico a los 3 meses de evaluación según tipo de tratamiento.

\begin{tabular}{|c|c|c|c|c|c|c|c|}
\hline & & \multicolumn{2}{|c|}{$\begin{array}{c}\text { Éxito } \\
\text { (sin lesión } \\
\text { aparente) }\end{array}$} & \multicolumn{2}{|c|}{$\begin{array}{c}\text { Fracaso } \\
\text { (lesión } \\
\text { evidente) }\end{array}$} & \multicolumn{2}{|c|}{ Total } \\
\hline & & $\mathbf{n}$ & $\%$ & $\mathbf{n}$ & $\%$ & $\mathbf{n}$ & $\%$ \\
\hline \multirow[t]{2}{*}{ Tipo de tratamiento } & CTZ & 20 & 100 & 0 & 0 & 20 & 50 \\
\hline & Formocresol & 18 & 90 & 2 & 10 & 20 & 50 \\
\hline Total & & 38 & 95 & 2 & 5 & 40 & 100 \\
\hline
\end{tabular}

Test de Fisher: 0,243 ( $p<0,05)$.

Tabla III. Comparación del comportamiento clínico y radiográfico a los 3 meses de evaluación según tipo de tratamiento.

\begin{tabular}{llcccc}
\hline & & $\begin{array}{c}\text { Éxito } \\
\text { Clínico }\end{array}$ & $\begin{array}{l}\text { Fracaso } \\
\text { Clínico }\end{array}$ & $\begin{array}{l}\text { Fracaso } \\
\text { Radiográfico }\end{array}$ & $\begin{array}{l}\text { Fracaso Clínico } \\
\text { y Radiográfico }\end{array}$ \\
\hline \multirow{2}{*}{$\begin{array}{l}\text { Tipo de tratamiento } \\
\text { Total }\end{array}$} & CTZ & 14 & 6 & 0 & 0 \\
& Formocresol & 15 & 5 & 2 & 2 \\
\hline
\end{tabular}

Test de Fisher: 0,424 $(p<0,05)$

Tabla IV. Comportamiento clínico a los 6 meses de evaluación según tipo de tratamiento

\begin{tabular}{llcccccc}
\hline & & \multicolumn{2}{c}{ Éxito } & \multicolumn{2}{c}{ Fracaso } & \multicolumn{2}{c}{ Total } \\
& & $\mathbf{n}$ & $\%$ & $\mathbf{n}$ & $\%$ & $\mathbf{n}$ & $\%$ \\
\hline Tipo de tratamiento & CTZ & 16 & 80 & 4 & 20 & 20 & 50 \\
& Formocresol & 17 & 85 & 3 & 15 & 20 & 50 \\
Total & & 33 & 82,5 & 7 & 17,5 & 40 & 100 \\
\hline
\end{tabular}

Test de Fisher: 0,502 $(p<0,05)$. 
LUENGO, F. J.; RAMOS, M. A.; HERNÁNDEZ, M. M. E.; DÍAZ, R. C. Y.; MEDRANO, L. E. C. \& TOSCANO, G. I. Efectividad clínica y radiográfica de la pasta antibiótica CTZ en pulpotomías de molares primarios. Ensayo clinico aleatorio controlado. Int. J. Odontostomat., 10(3):425-431, 2016.

Tabla V. Comportamiento radiográfico a los 6 meses de evaluación según tipo de tratamiento.

\begin{tabular}{llllllll}
\hline & & \multicolumn{2}{c}{ Éxito } & \multicolumn{2}{c}{ Fracaso } & \multicolumn{2}{c}{ Total } \\
& & $\mathbf{n}$ & $\%$ & $\mathbf{n}$ & $\%$ & $\mathbf{n}$ & $\%$ \\
\hline Tipo de tratamiento & CTZ & 16 & 80 & 4 & 20 & 20 & 50 \\
& Formocresol & 13 & 65 & 7 & 35 & 20 & 50 \\
Total & & 29 & 72,5 & 11 & 27,5 & 40 & 100 \\
\hline
\end{tabular}

Test de Fisher: $0,240(p<0,05)$.

Tabla VI. Comparación del comportamiento clínico y radiográfico a los 6 meses de evaluación según tipo de tratamiento.

\begin{tabular}{llccc}
\hline & $\begin{array}{l}\text { Éxito } \\
\text { Clínico }\end{array}$ & $\begin{array}{c}\text { Fracaso } \\
\text { Clínico }\end{array}$ & $\begin{array}{l}\text { Fracaso } \\
\text { Radiográfico }\end{array}$ & $\begin{array}{l}\text { Fracaso Clínico } \\
\text { y Radiográfico }\end{array}$ \\
\cline { 2 - 5 } Tipo de tratamiento & CTZ & 16 & 4 & 4 \\
& Formocreso & 17 & 3 & 7 \\
Total & 33 & 7 & 11 & 2 \\
\hline
\end{tabular}

Test de Fisher: 0,854 ( $p<0,05)$.

\section{DISCUSIÓN}

La Terapia pulpar en dientes primarios resulta en ocasiones difícil de realizar por algunas razones como, la dificultad que se presenta para el manejo de la conducta del infante, las diferencias en la morfología de los dientes, la complejidad de los conductos radiculares, poca certeza del proceso reabsorción de las raíces, y las dificultades para la colocación del material en el interior de los conductos radiculares (Nanda et al., 2014).

Las técnicas de tratamiento propuestas dentro de esta terapia son la pulpotomía y la pulpectomía. La pulpotomía, se considera un tratamiento conservador para revertir el proceso inflamatorio de la pulpa, limitándose sólo a la remoción de ésta dentro de la cámara pulpar. Por otro lado, la pulpectomía es un tratamiento radical empleado cuando la pulpa se encuentra en estado irreversible patológico, donde se remueve todo el tejido pulpar, tanto coronal como radicular, eliminando todas las bacterias que allí se encuentren (Nanda et al.; Bruno et al., 2006).

En situaciones en las que progresa rápidamente el proceso patológico de la caries dental, ésta puede evolucionar produciendo afectación pulpar, abscesos y necrosis, recomendándose en muchos de los casos la realización de la pulpectomía. Sin embargo, se debe considerar que los dientes deciduos presentan estructuras y reacciones tisulares diferentes que las que tienen los dientes permanentes, lo que hace que algunas de formas de tratamiento sean menos adecuadas. Así mismo, cuando se estudia la anatomía de los dientes primarios, se observa que presentan conductos radiculares complejos, irregulares, estrechos y conductos accesorios, lo que dificulta una correcta manipulación de los mismos cuando se realiza procedimientos pulpares como la pulpectomía (Bruno et al.).

El uso de fármacos antibacteriales para tratar lesiones endodónticas es uno de los procedimientos clínicos que pueden ser utilizados para la esterilización de las lesiones. En este sentido, el uso de antibióticos de manera sistémica y tópica, ha sido utilizado en la medicina y la odontología durante años, especialmente para tratar procesos infecciosos asociados a dientes con afección pulpar. No obstante, se ha reportado que cuando se administra un antibiótico de forma sistémica, solo una pequeña parte de la concentración del fármaco alcanza el conducto radicular, lo que lo hace poco benéfico. Frente a lo anterior, la aplicación de antibióticos locales permite administrar concentraciones sustancialmente más elevadas y evitar complicaciones sistémicas (Hoelscher et al, 2006).

El manejo de la evidencia anteriormente descrita, ha permitido que desde varios años en la Clínica Integral de la Especialidad en Odontopediatría de la Universidad Autónoma de Zacatecas, se instaure el tratamiento endodóntico no instrumentado en dientes primarios, mediante uso de una pasta antibiótica que contiene la mezcla de fármacos antibacterianos para la desinfección de órganos dentales con afectación pulpar y pronostico dudoso. 
LUENGO, F. J.; RAMOS, M. A.; HERNÁNDEZ, M. M. E.; DÍAZ, R. C. Y.; MEDRANO, L. E. C. \& TOSCANO, G. I. Efectividad clínica y radiográfica de la pasta antibiótica CTZ en pulpotomías de molares primarios. Ensayo clinico aleatorio controlado. Int. J. Odontostomat., 10(3):425-431, 2016.

Este enfoque biológico se considera innovador, debido a que no se requiere de instrumentación mecánica, lo que evita una mayor ampliación de los conductos radiculares, irritación innecesaria de los tejidos periapicales, disminuyendo los tiempos operatorios (realizándose en una sola cita), pudiendo ser aplicado de manera satisfactoria en niños pequeños durante la primera infancia (Nanda et al.).

En el desarrollo del presente estudio, la principal dificultad encontrada fue la falta de literatura concluyente sobre el tema, especialmente cuando se realiza en pulpotomías vitales, por lo que fue necesario emplear investigaciones In Vitro, In Vivo y en pulpas necróticas; realizando homologías donde se ha demostrado su efectividad y biocompatibilidad, los cuales se consideran parámetros de referencia principal para el estudio de las propiedades biológicas, en especial para materiales que estarán en boca durante un largo período de tiempo.

En los seres humanos, los efectos biológicos se evalúan por la respuesta de la pulpa y de los tejidos periapicales. El método de evaluación de la respuesta es por signos y síntomas y por la evaluación radiográfica (Fernández et al., 2006). Éste estudio comparó de forma clínica y radiográfica tratamientos de pulpotomías realizados con la pasta antibiótica CTZ versus la técnica convencional con formocresol en molares primarios, durante un periodo de 3 y 6 meses.

Durante las evaluaciones, nuestros resultados no mostraron diferencias estadísticamente significativas en las tasas de éxito y fracaso entre los grupos de estudio. Sin embargo, en la evaluación de los 6 meses se pudo observar una eficiencia clínica y radiográfica del $85 \%$ y $65 \%$ respectivamente, en el grupo de formocresol. Para estos resultados, habría que recordar que el formocresol es un poderoso antiséptico que actúa tanto por contacto como a distancia (Fernández et al.), el cual ha sido avalado como el "Gold Estándar" para tratamientos de pulpotomías en molares primarios, (Peng, 2007; Durán Comparán, 2008) mostrando altas tasas de éxito clínico.

De igual manera, en el grupo de molares que recibieron la aplicación de la pasta CTZ obtuvieron altas tasas de eficiencia clínica y radiográfica que oscilan en un $80 \%$. Estos resultados coinciden con Takushige et al. $(2008,2009)$, los cuales observaron una buena evolución clínica del $95 \%$ en molares tratados con la técnica no instrumentada y con la aplica- ción de una pasta antibiótica. Así mismo, concuerdan con Nakornchai et al. (2010), los cuales reportaron 100 $\%$ de éxito clínico y $76 \%$ de éxito radiográfico. No obstante, difieren levemente, desde el punto de vista clínico, de los valores reportados por Trairatvorakul \& Detsomboonrat (2012); los cuales muestran $75 \%$ de éxito clínico y un bajo éxito radiográfico de un $36,7 \%$.

Los resultados encontrados en esta investigación sugieren que el éxito clínico de la pasta CTZ, podría atribuirse al hecho de que en los dientes primarios, la presencia de conductos accesorios, porosidad y permeabilidad en la región del piso pulpar indica una probable conexión entre el tejido pulpar y los tejidos periodontales. La combinación de fármacos antibacterianos puede disiparse fácilmente a través de estas regiones e inducir una zona estéril (Trairatvorakul \& Detsomboonrat). Otro factor importante, es la realización de la antisepsia en la zona y colocación de la pasta que con su acción bacteriostática origina que la carga bacteriana se reduzca, ya que, desde su colocación modifica la microflora existente haciendo que el número de los microorganismos disminuyan modificando su patogenicidad (Pérez et al., 2012).

Finalmente, la pulpotomía con CTZ es una técnica mínimamente invasiva y no amerita de limado ni ensanchado de los conductos radiculares, permitiendo la culminación del procedimiento en una sola cita, facilitando un mejor manejo de la conducta del paciente pediátrico, ya que, disminuye los tiempos operatorios.

LUENGO, F. J.; RAMOS, M. A.; HERNÁNDEZ, M. M. E.; DÍAZ, R. C. Y.; MEDRANO, L. E. C. \& TOSCANO, G. I. Clinical and radiographic effectiveness of antibiotic paste CTZ in primary molars pulpotomy. Ramdomized controlled clinical trial. Int. J. Odontostomat., 10(3):425-431, 2016.

ABSTRACT: The objective of this study to evaluate the clinical and radiographic effectiveness of antibiotic paste CTZ in pulpotomy of primary molars. A randomized controlled clinical trial was performed, in 40 molars of 40 patients aged 3 to 6 years. Were selected and randomly assigned to two groups: Formocresol $(n=20)$, CTZ (ChloramphenicolTetracycline-Zinc Oxide Eugenol, $n=20$ ). The teeth were restored with glass ionomer and performed steel metal crowns. Clinical and radiographic evaluation procedure was performed at 3 and 6 months. SPSS v.17 program for data analysis, and application of the Fisher exact test was used 5 $\%$. The clinical behavior at 3 months showed $75 \%$ success rate for molars treated with formocresol and $70 \%$ for the CTZ. Radiographically $90 \%$ success rate for the group with formocresol and $100 \%$ for the CTZ paste was obtained. At 6 months formocresol clinical success was $85 \%$ and $80 \%$ 
CTZ. Radiographic success was shown $65 \%$ for those treated with formocresol and $80 \%$ for the CTZ ( $p>0.05)$. No significant differences in the results shown were observed. CTZ paste is an alternative in the treatment of pulpotomy of molars. It provides an antimicrobial effect, stabilizing the process of root resorption, without causing damage to the permanent tooth formation.

KEY WORDS: primary, molars, pulpotomy, formocresol, CTZ.

\section{REFERENCIAS BIBLIOGRÁFICAS}

Bruno, G. B.; Menezes, V. A.; Bruno, J. A.; Almeida, M. W. \& Viana, G. S. B. Avaliação hematológicas e bioquímicas do sangue de cães submetidas a pulpotomias com cimento de antibiótico. Rev. Odontol. U. N. E. S. P., 35(3):125-33, 2006.

Durán Comparán, B. E. Eficacia clínica del formocresol en comparación con el hidróxido de calcio en pulpotomías de dientes primarios: Reporte preliminar. Rev. A. D. M., 65(3):117-20, 2008.

Fernández, A. V.; Giro, E. M. A. \& Costa, C. A. S. Resposta dos tecidos periapicais de dentes de cães com necrose pulpar $e$ reação periapical crônica ao tratamento endodôntico utilizando diferentes pastas obturadoras. Rev. Odontol. U. N. E. S. P., 35(1):29-39, 2006.

González-Núñez, D.; Trejo-Quiroz, P.; De León-Torres, C. \& Carmona-Ruiz, D. Técnica de endodoncia no instrumentada mediante el uso de la pasta CTZ. Rev. Estomat., 18(2):27-32, 2010 .

Hoelscher, A. A.; Bahcall, J. K. \& Maki, J. S. In vitro evaluation of the antimicrobial effects of a root canal sealer-antibiotic combination against Enterococcus faecalis. J. Endod., 32(2):145-7, 2006.

Sousa, P. M.; Duarte, R. C. \& de Sousa, S. A. Clinical and radiographic monitoring of primary teeth submitted to pulp therapy with CTZ paste. Pesqui. Bras. Odontopediatria Clin. Integr., 14(3):56-68, 2014.

Molina-Frechero, N.; Durán-Merino, D.; Castañeda-Castaneira, E. \& Juárez-López, M. L. A. La caries y su relación con la higiene oral en preescolares mexicanos. Gac. Med. Mex., 151:485-90, 2015.

Nakornchai, S.; Banditsing, P. \& Visetratana, N. Clinical evaluation of $3 \mathrm{Mix}$ and Vitapex as treatment options for pulpally involved primary molars. Int. J. Paediatr. Dent., 20(3):214-21, 2010.

Nanda, R.; Koul, M.; Srivastava, S.; Upadhyay, V. \& Dwivedi, R. Clinical evaluation of $3 \mathrm{Mix}$ and Other Mix in non-instrumental endodontic treatment of necrosed primary teeth. J. Oral Biol. Craniofac. Res., 4(2):114-9, 2014

de Oliveira, M. A. C. \& Costa, L. R. R. S. Desempenho clínico de pulpotomías com pasta CTZ en molares decíduos: Estudio retrospectivo. Robrac., 15(40):37-45, 2006.
Passos, I. A.; Melo, J. M. \& Moreira, P. V. L. Utilização da pasta CTZ em dente decíduo com necrose pulpar: relato de caso. Odontol. Clin. Cient., 7(1):63-5, 2008.

Peng, L.; Ye, L.; Guo, X.; Tan, H.; Zhou, X.; Wang, C. \& Li, R Evaluation of formocresol versus ferric sulphate primary molar pulpotomy: a systematic review and meta-analysis. Int. Endod. J., 40(10):751-7, 2007.

Pérez, H. P. E.; Curioca, R. S. A. \& Retana, U. R. Efectividad terapéutica de la pasta CTZ vs. biomecánica convencional en pulpa necrótica de escolares de 4-8 años. Odont. Pediatr. Act., 1(3):28-36, 2012.

Perona, G. \& Mungi, S. Tratamiento endodóntico no instrumentado en dientes deciduos. Rev. Odontopediatr. Latinoam., 4(1):5364, 2014

Pinky, C.; Shashibhuhshan, K. K. \& Subbareddy, V. V. Endodontic treatment of necrosed primary teeth using two different combinations of antibacterial drugs: an in vivo study. J. Indian Soc. Pedod. Prev. Dent., 29(2):121-7, 2011.

Subramaniam, P. \& Gilhotra, K. Endoflas, zinc oxide eugenol and metapex as root canal filling materials in primary molars--a comparative clinical study. J. Clin. Pediatr. Dent., 35(4):365-70, 2011.

Takushige, T.; Cruz, V. E.; Moral, A. M. \& Hoshino, E. Non-surgical treatment of pulpitis, including those with history of spontaneous pain, using a combination of antibacterial drugs. J. LSTR Ther., 7:1-5, 2008.

Takushige, T.; Hataoka, H.; Ando, M. \& Hoshino, E. Endodontic retreatment using 3Mix- MP without removal of previous root canal obturation J. LSTR Ther., 8:3-7, 2009.

Trairatvorakul, C. \& Detsomboonrat, P. Success rates of a mixture of ciprofloxacin, metronidazole, and minocycline antibiotics used in the non-instrumentation endodontic treatment of mandibular primary molars with carious pulpal involvement. Int. J. Paediatr. Dent., 22(3):217-27, 2012.

World Medical Association. World Medical Association Declaration of Helsinki: ethical principles for medical research involving human subjects. JAMA, 310(20):2191-4, 2013.

Dirección para correspondencia

Jesús Alberto Luengo Fereira

Programa de Especialidad en Odontopediatría

Unidad Académica de Odontología

Universidad Autónoma de Zacatecas (UAZ)

Carretera a la Bufa S/N,

Colonia Centro Zacatecas

Código Postal 98000

Zac.

MÉXICO

Received: 25-04-2016

Accepted: 03-08-2016

Email: jluengofereira@gmail.com 